Mots. Les langages du politique

\title{
De l'AMI aux ABI La libre circulation des capitaux en marche
}

From ami to abi. the forward march of free circulation of capital

Del ami a los abi. la libre marcha de la circulacion de los capitales

Jean-Claude Deroubaix

\section{OpenEdition}

Journals

Édition électronique

URL : https://journals.openedition.org/mots/7543

DOI : $10.4000 /$ mots. 7543

ISSN : 1960-6001

Éditeur

ENS Éditions

Édition imprimée

Date de publication : 1 mars 2003

Pagination : 11-22

ISBN : 2-84788-027-5

ISSN : 0243-6450

\section{Référence électronique}

Jean-Claude Deroubaix, « De l'AMI aux ABI La libre circulation des capitaux en marche », Mots. Les

langages du politique [En ligne], 71 | 2003, mis en ligne le 05 mai 2008, consulté le 23 avril 2022. URL http://journals.openedition.org/mots/7543 ; DOl : https://doi.org/10.4000/mots.7543

\section{(C) ENS Éditions}




\section{De 1'AMI aux $A B I$ La libre circulation des capitaux en marche}

Les Accords bilatéraux de protection et de promotion des investissements (ABI) sont les outils discrets mis en œuvre par le monde des affaires et les gouvernements pour faciliter une circulation mondiale des capitaux sans contraintes. Ces accords, signés entre deux États souverains, offrent une protection mutuelle aux investissements faits par une personne (morale ou physique) d'un État dans l'autre. Ils sont signés en grand nombre depuis les années 1980, la Banque mondiale estime qu'environ 2000 de ces accords sont actuellement en vigueur. À l'origine (dans les années 1950), ils étaient conclus entre un pays développé et un pays que l'on désignait alors en voie de développement - on dit maintenant pays du Sud ou pays moins avancé. Désormais, nombre de ces ABI sont établis entre pays du Sud.

Depuis 1976, on assiste à des tentatives de mises en place d'un système global qui garantirait la libre circulation des capitaux, afin de conforter la suprématie de la société de marché sur tout autre système de société. La première tentative d'Accord multilatéral sur les investissements (AMI) de 1976, engagée sous l'égide de l'OCDE ${ }^{1}$, s'est soldée par un échec. Une seconde tentative, menée dans le plus grand secret à l'OCDE encore, a finalement été abandonnée en 1998 sous la pression des $\mathrm{ONG}^{2}$ et des pays en voie de développement qui avaient été tenus à l'écart des négociations.

Un nouvel essai est en cours dans le cadre de l'OMC $^{3}$, mais il ne

- GRAID, ULB Bruxelles ; SYLED, Paris 3.

1. Organisation de coopération et de développement économiques. Cette organisation intergouvernementale a son siège à Paris (sigle en anglais : OECD) et comporte 29 pays membres (les USA, les membres de l'Union européenne, la Suisse, la Turquie, le Japon, etc.).

2. Organisation non-gouvernementale : en général désigne les organisations volontaires qui poursuivent des buts non lucratifs, sociaux, humanitaires, écologiques, et dont l'action se situe au plan international.

3. L'Organisation mondiale du commerce créée le $1^{\text {er }}$ janvier 1995 pour supprimer les obstacles au commerce. Elle comporte aujourd'hui quelque 140 pays membres. En anglais : WTO. 
concerne que les investissements liés au commerce (Accord général sur le commerce et les services), une grande part de l'investissement mondial reste donc hors de son champ.

Il n'est, dès lors, pas étrange que soit négociée et signée, à titre compensatoire, une théorie d'accords bilatéraux. Accords qui, en maillant le monde, sont en train de construire la base d'un futur AMI. La question est de savoir si un tel maillage n'est finalement pas plus efficace pour assurer les objectifs de libéralisation des capitaux que la négociation centralisée d'un AMI soumis aux feux de l'actualité.

La négociation de chacun de ces accords est évidemment centrée sur les termes de l'accord, sur le vocabulaire et les définitions qu'il comporte. C'est à cet aspect-là de la question que nous nous arrêterons dans cet article, renvoyant à l'étude du GRAID ${ }^{4}$ sur les accords bilatéraux pour les questions de sociologie politique que soulève la multiplication de ces conventions.

\section{La liberté d'user de son propre vocabulaire}

La liberté de négocier commence avec celle d'user de son propre vocabulaire. Or cette liberté d'user ou non de son propre vocabulaire, de ses propres définitions ne peut être garantie aux deux parties, puisque c'est précisément sur cela que porte la négociation. En outre, nul ne négocie «en l'air», sans partir d'un texte de base qui subira les amendements de chacune des parties. Dans une négociation, quel que soit son objet, le choix du texte de base est primordial parce qu'il va donner à l'une des parties un avantage certain, celui d'imposer avec et par son vocabulaire un point de vue général, un cadre qui délimitera la discussion.

Or, la fiction d'accords bilatéraux négociés librement entre deux parties égales parce que souveraines ne résiste pas à la réalité de la constatation d'une forte parenté juridique et politique (donc lexicale) entre tous les accords bilatéraux censément indépendants. Cette parenté dans les clauses, mais aussi dans les termes utilisés et dans leur définition, découle du fait qu'en réalité, sous-jacent à ces accords existe un «draft» ${ }^{5}$, un

4. Groupe de recherche sur les acteurs internationaux et leurs discours.

5. «Developed countries made negotiation offers to these developing countries individually. These offers were made on the basis of the provisions in the draft OECD convention for the protection of foreign assets. The draft convention was thus promoted de facto to the rank of a model treaty on protection of investments, which the OECD members came to rely upon in their bilateral dealings with non-OECD members», Jacques Juilliard, «Bilate- 
brouillon, rédigé dans les années 1980 à l'OCDE, qui sert de modèle aux textes que proposent les États occidentaux lorsque s'ouvrent des négociations bilatérales. M. Louis Michel $^{6}$, ministre belge des Relations extérieures, s'est ainsi réjoui lors de la présentation de l'accord entre 1'Union économique belgo-luxembourgeoise et les Comores (UEBL : en cette matière les deux États agissent toujours conjointement) de ce que non seulement le texte de l'UEBL avait été accepté comme point de départ mais qu'il se retrouvait quasiment tel quel à l'arrivée. Polybe disait : « Le commencement est la moitié du tout», mais dans une négociation, c'est bien plus que la moitié d'imposer sa proposition comme point de départ. On impose en même temps son vocabulaire, ce qui est loin d'être innocent. C'est ce que nous tenterons de montrer sur quelques exemples dans cette contribution.

\section{Bilatéral, mutuel et réciproque}

Les accords sont bilatéraux et prévoient une protection mutuelle ou réciproque des investissements. Cela implique une égalité des droits des deux parties. Pourtant dans les discussions sur la nécessité de ce genre d'accords, les ministres et les hauts fonctionnaires occidentaux insistent sur le fait que les pays "pauvres» sont demandeurs de capitaux et qu'il s'agit donc bien de donner aux pays exportateurs de capitaux des garanties. L'asymétrie est évidente. Avant l'invention de l'accord bilatéral, les États puissants imposaient leurs conditions dans des traités dits de navigation et d'amitié, dans lesquels les obligations n'étaient pas réciproques, laissant à nu la domination coloniale. L'astuce du recours au vocabulaire des obligations mutuelles et réciproques permet de cacher l'inégalité de fait de ces obligations.

ral investment treaties in the context of investment law» dans OECD-OCDE seminar on Investment compact regional roundtable on bilateral investment treaties for the protection and promotion of foreign investment in South East Europe, 28-29 mai 2001, Dubrovnik, Croatie. [Les pays développés font des offres de négociations à ces pays en voie de développement individuellement. Ces offres sont faites sur la base des éléments de l'esquisse de convention de l'OCDE pour la protection des investissements. Cette esquisse de convention est donc de facto promue au rang de modèle de traité sur la protection des investissements sur lequel les pays membres de l'OCDE peuvent s'appuyer dans leurs négociations bilatérales avec les pays qui ne sont pas membres de l'OCDE. Traduction JCD].

6. «Les négociations se sont basées au départ sur le texte de base de l'UEBL, version 
L'aspect bilatéral de ces négociations est évidemment battu en brèche par la multiplication réticulaire de ces accords très semblables, mais aussi par les dispositions techniques prises pour leurs conclusions. Ainsi la $\mathrm{CNUCED}^{7}$ a-t-elle inventé le round multilatéral de négociations bilatérales.

Encore une fois cédons la parole au ministre Michel à propos de l'accord UEBL-Comores :

En janvier 2001, la CNUCED à Genève a pris l'initiative de faciliter la conclusion d'accords d'investissement entre pays moins avancés (PMA) ou entre pays moins avancés et pays industrialisés en regroupant ces accords et en organisant les négociations au siège de la CNUCED à Genève. De cette manière, le «profil économique» de ces pays moins avancés se trouve mis en évidence de manière plus efficace et plus rapide. Le grand avantage de cette procédure réside dans le fait que les négociations avec une série de pays moins avancés se déroulent en un même endroit, ce qui a pour effet de réduire considérablement la durée et le cout des négociations ${ }^{8}$.

Les négociations n'ont pas seulement lieu au siège de la CNUCED à Genève, mais aussi au Pérou ou au Japon. Le bilatéral devient, dans ce cas, une sous-espèce du multilatéral. Ce contexte globalisant prend appui sur le fait qu'il existe une école de formation à la conduite de ce type d'accords. En 2001 et en 2002, cette formation a été organisée à Alexandrie ${ }^{9}$ sous l'égide de la CNUCED, où de futurs négociateurs reçoivent des cours et participent à des simulations de négociation d'accords sur l'investissement. Ils obtiennent même un diplôme. Mais ce dont ils ont surtout bénéficié, c'est d'une imprégnation de la culture de la protection de l'investissement.

française, qui A ÉTÉ ACCEPTÉE DANS SA TOTALITÉ par le chef de la délégation des Comores, à l'exception de la définition du territoire des deux Parties contractantes, tant l'UEBL que les Comores ayant voulu maintenir leur propre texte (art. 1, § 4, (a) et (b)) », dans Exposé des motifs du Projet de loi portant assentiment à l'Accord entre l'Union économique belgo-luxembourgeoise et la République fédérale islamique des Comores concernant l'encouragement et la protection réciproques des investissements, fait à Bruxelles le 18 mai 2001, document 2-1070/1, Sénat de Belgique, Session de 2001-2002, 12 mars 2002. Les majuscules sont dans le rapport.

7. La CNUCED, Conférence des Nations unies pour le commerce et le développement, en anglais UNCTAD, est un organe spécialisé de l'ONU.

8. Voir aussi le document UNCTAD/ITE/IIA/2 de la CNUCED intitulé : Bilateral investment treaties (1959-1999), New York et Genève, 2000, pages 3, 4, 5.

9. Centre René-Jean Dupuy de l'Université Senghor d'Alexandrie. 


\section{Investissement}

Chacun de ces accords comporte une clause extrêmement importante sur la définition de ce qu'est un investissement, en somme la délimitation de ce qui doit être protégé. La formule reprise dans le texte de l'accord UEBL-Comores, qui, de l'aveu même du ministre belge, est identique au modèle que tente d'imposer l'UEBL, est celle-ci :

2. Le terme «investissements » désigne tout élément d'actif quelconque et tout apport direct ou indirect en numéraire, en nature ou en services, investi ou réinvesti dans tout secteur d'activité économique, quel qu'il soit.

Sont considérés notamment, mais non exclusivement, comme des investissements au sens du présent Accord:

a) les biens meubles et immeubles ainsi que tous autres droits réels tels que hypothèques, privilèges, gages, usufruit et droits similaires ;

b) les actions, parts sociales et toutes autres formes de participations, même minoritaires ou indirectes, dans le capital de sociétés constituées sur le territoire de l'une des Parties contractantes ;

c) les obligations, créances et droits à toutes prestations ayant une valeur économique ;

d) les droits d'auteur, les droits de propriété industrielle, les procédés techniques, les noms déposés et le fonds de commerce ;

e) les concessions de droit public ou contractuelles, notamment celles relatives à la prospection, la culture, l'extraction ou l'exploitation de ressources naturelles. Aucune modification de la forme juridique dans laquelle les avoirs et capitaux ont été investis ou réinvestis n'affectera leur qualité d'investissements au sens du présent Accord.

3. Le terme «revenus» désigne les sommes produites par un investissement et notamment, mais non exclusivement, les bénéfices, intérêts, accroissements de capital, dividendes, royalties ou indemnités.

Dans le projet d'accord multilatéral (AMI) se trouvait par contre la définition suivante :

2. On entend par «investissement»:

Tout type d'actif détenu ou contrôlé, directement ou indirectement, par un investisseur, notamment :

(i) une entreprise (personne morale ou autre entité constituée ou organisée selon le droit applicable d'une partie contractante, avec ou sans but lucratif, privée ou appartenant à une autorité publique ou contrôlée par elle, y compris une société de capitaux, fiducie, société de personnes, entreprise individuelle, succursale, co-entreprise, association ou organisation) ;

(ii) les actions, parts de capital ou autres formes de participation au capital d'une entreprise et les droits en découlant ; 
(iii) les obligations, titres d'emprunt, prêts et autres formes de créance et les droits en découlant ;

(iv) les droits au titre de contrats, notamment les contrats clés en main et les contrats de construction, de gestion, de production ou de partage des recettes ;

(v) les créances monétaires et les droits à prestations ;

(vi) les droits de propriété intellectuelle ;

(vii) les droits conférés par la loi tels que les concessions, licences, autorisations et permis ;

(viii) tout autre bien corporel ou incorporel, meuble ou immeuble, et tous droits connexes de propriété tels que location, hypothèque, privilège et gage.

La première remarque qui vient en lisant ces définitions est qu'elles se caractérisent toutes les deux par l'amplitude du champ couvert. Les listes de types d'investissements ne sont que des exemples, et la liste n'est pas exhaustive. Les deux listes peuvent donc différer légèrement sans mettre en cause l'identité des définitions : « tout» type d'actif, détenu ou contrôlé « directement» ou «indirectement» par un investisseur étranger (ou de l'autre partie).

Investissement est dans le sens que lui donnent ces accords, un mot neuf ${ }^{10}$ en français, calque de investment en anglais. En effet, pour Littré, investissement renvoie au seul sens "action d'entourer une place, une ville, et d'en couper les communications », investir comporte en outre la signification de mettre quelqu'un en possession d'un pouvoir (voir investiture), définitions proches de celles des dictionnaires de l'Académie de 1694 à 1878 et du Dictionnaire de Furetière. Par contre, ces dictionnaires désignent l'action de disposer de son argent pour le faire fructifier et produire de l'intérêt par le mot placement. L'économie politique keynésienne des années 1940 à 1990 fait encore une distinction importante entre placement et investissement, le premier terme désignant l'acte d'épargne, purement financier, le second la mise en œuvre d'un capital en vue de la production de biens ${ }^{11}$.

En confondant les deux actions sous le seul terme d'investissement les promoteurs du modèle laissent supposer qu'effectivement les flux financiers protégés par l'accord sont des investissements au sens keynésien du terme.

10. Dans Le Robert, Dictionnaire historique de la langue française, on situe autour de 1920 l'usage d'investir (1922), et d'investissement (1924), au sens anglais «d'employer des capitaux dans une entreprise». Quant à l'usage d'investir comme substitut de placer, Le Robert se contente d'un renvoi non daté à une extension de sens.

11. Il peut être renvoyé à tout bon manuel d'économie politique à ce sujet. Par exemple 
Cette confusion n'est pas fortuite. Les documents de l'OCDE, de l'OMC ou de la CNUCED qui promeuvent la signature de tels accords mettent systématiquement en évidence une autre définition de l'investissement : «l'investissement direct doit être durable et productif, l'investisseur doit assurer une présence réelle dans le pays hôte». Or cette définition ne ressemble pas à celle qui s'impose dans les textes contraignants comme les accords de protection de l'investissement ou les accords de libre-échange. Ces conditions, "être durable, productif et réel», ne sont pas reprises dans les accords bilatéraux qui laissent ainsi place à des mouvements de capitaux qui n'ont pas nécessairement un caractère d'investissement durable. De fait, l'essentiel des investissements directs étrangers que répertorie chaque année l'OCDE est composé d'acquisition d'entreprises étrangères et non de création d'activités nouvelles.

Il y a donc jeu de mots. Mais un jeu dangereux : puisque sous l'apparence de la mobilisation de capitaux pour le développement, c'est la circulation générale de tous les types de capitaux qui est organisée.

\section{National}

Nous avons déjà fait allusion à l'aspect réticulaire des ABI. Nous le retrouverons avec l'emploi du mot national qui est fait dans ce genre d'accords.

En effet, ces conventions entre États ont pour objectif de protéger les investissements faits par des nationaux d'une partie sur le territoire de l'autre partie. Ces nationaux peuvent être des personnes physiques possédant la nationalité d'une des parties, mais qu'en est-il des personnes morales, des sociétés, des compagnies ? Quel sera le critère de nationalité? En général, le choix est fait de la déterminer par le siège social de l'entreprise ou de la société en question ${ }^{12}$. Cela a donc comme conséquence qu'un État $\mathrm{X}$ va protéger les investissements, sur le territoire de l'État Y, d'une société A dont le siège social se trouve en X, mais dont rien n'est spécifié quant à la nationalité des capitaux de cette société, ni de son centre de contrôle. Comme le rappelle bien Madeleine Albright, secrétaire d'État des États-Unis d'Amérique, «The broad nature of the definition of «investment» and «company» of a Party means that investments can be

à Yves de Wasseige, 1970, Les mécanismes de l'économie moderne, Bruxelles, EVO éditeur, p. 96, 97.

12. «Les "sociétés", c'est-à-dire toute personne morale constituée conformément à la 
covered by the Treaty even if ultimate control lies with non-Party nationals ${ }^{13}$ »).

Il est, dès lors, évident qu'il ne s'agit plus d'ABI strictement indépendants, les sociétés multinationales pouvant parfaitement opter pour une stratégie de protection de leurs investissements en délocalisant les sièges de leurs filiales selon les besoins. Derrière la notion de «national» ne se trouvent plus que des stratégies privées ${ }^{14}$ : le capital n'a pas de nationalité.

La deuxième référence à national rencontrée dans ces textes apparait dans le syntagme : traitement national. Il s'agit d'une clause qui garantit à l'investisseur étranger qu'il sera traité en tous points comme l'investisseur national. Il est donc superflu de lui imposer quelque contrainte supplémentaire que ce soit. Or, cette prétendue égalité n'existe pas dans les faits. En effet, si sur le plan des contraintes une certaine égalité est établie, ce n'est pas le cas sur le plan des privilèges accordés à l'investisseur étranger : le droit de retirer ses avoirs en tout temps, la possibilité d'être indemnisé en cas de troubles sociaux, le recours à un tribunal arbitral international en lieu et place des tribunaux locaux s'il le souhaite.

\section{Juge et arbitre}

M. Pascal Lamy, commissaire européen au commerce, parlant de la sécurisation des investissements dans le monde, écrit :

Puis viennent successivement le tour des liens entre commerce et investissement afin de sécuriser les flux d'investissements et d'en faire davantage profiter les pays en développement [...]. Ces trois sujets étaient importants pour nous dans la perspective d'un processus de mondialisation encadré par des règles plus solides. Ils sont acceptés. Ces règles conduiront à la constitution ou

législation du Royaume de Belgique, ou du Grand-Duché de Luxembourg ou de la République Fédérale Islamique des Comores et ayant son siège social sur le territoire du Royaume de Belgique, ou du Grand-Duché de Luxembourg ou de la République Fédérale Islamique des Comores respectivement» (dans Accord UEBL-Comores).

13. In Investment treaty with Lithuania, Letter of Submittal, Department of State, Washington, August 9, 2000 (document : Treaty Doc. 106-42, 106 ${ }^{\text {th }}$ session Congress, Senate, Washington, 2000) [Du fait que la définition des mots investissement et société d'une des Parties est large, les investissements peuvent être couverts même si la société qui investit est sous le contrôle ultime de personnes qui ne sont des ressortissants d'aucune des deux Parties. Trad. JCD].

14. Le Dr Bill Rosenberg, chercheur néo-zélandais de Action, Research and Education Network of Aotearoa, rappelle le cas récent de la société Aguas del Altuni - filiale de Inter- 
au renforcement d'autorités publiques appelées à juger des litiges de concurrence ou de marchés publics sur la base des mêmes principes, selon des procédures contradictoires et avec des possibilités de recours. Une avancée donc pour la règle de droit au détriment de l'arbitraire qui règne encore souvent ${ }^{15}$.

Il était alors à Doha, à l'assemblée générale de l'OMC, où commençait la négociation d'un accord général sur le commerce et les services comprenant un volet sur la protection des investissements.

Les autorités publiques auxquelles il fait référence sont déjà existantes : le CIRDI ${ }^{16}$ est l'organe le plus connu, avec, évidemment, celui du règlement des différends de l'OMC.

Les accords bilatéraux, tout comme l'AMI le proposait, autorisent un investisseur étranger à recourir à un tribunal d'arbitrage international s'il ne peut jouir en toute liberté de ses avoirs investis sur le territoire de l'autre partie contractante.

Encore une fois, au profit d'un glissement de sens, voilà élevés au rang de juges, investis d'une autorité publique, ceux que jusqu'ici on appelait des arbitres. Mieux, les voilà à même de donner un avis sur les juridictions nationales puisqu'ils sont chargés d'examiner si elles ont bien respecté les clauses des traités de protection de l'investissement. Ils ne doivent se soucier d'aucune autre législation, nationale ou internationale, si ce n'est de manière subsidiaire et toujours en tenant compte de l'objet premier de l'Accord à savoir la protection du capital. Ces «jugements» sont d'ailleurs toujours discrets ${ }^{17}$, et même souvent purement et simplement secrets.

national Waters ltd, société anglaise elle-même filiale de Bechtel Vorporation, société étatsunienne - qui a déplacé son siège des Iles Caïman vers les Pays-Bas pour bénéficier de la protection de l'accord bilatéral entre les Pays-Bas et la Bolivie alors qu'elle était en conflit avec la province de Cochabamba quant à la manière dont elle avait géré sa concession de distribution de l'eau (voir site http://www.arena.org.nz/billsand).

15. Pascal Lamy, 2002, L'Europe en première ligne, Paris, Le Seuil, p. 159.

16. Le Centre international de règlement des différends sur les investissements (en anglais ICSID) est un organe du groupe de la Banque mondiale. Son siège est à Genève. Il s'agit du seul organisme public (en tout cas intergouvernemental) qui organise des arbitrages internationaux.

17. Les arbitrages soumis au CIRDI bénéficient d'une publicité restreinte : ne sont diffusés que les noms des protagonistes, la composition du panel d'arbitres et les dates des différents actes de procédures. Le déroulement de l'arbitrage et les sentences arbitrales ne sont publiées que si les deux parties en cause donnent un accord explicite. Pour les autres organismes qui se chargent de l'arbitrage international, tout est secret. La Cour d'arbitrage internationale de Londres, organisme privé, en fait même un argument pour attirer les arbitrages en son sein. 
Sous l'apparence donc d'une convention qui institue un droit international et érige des institutions pour dire ce droit, nous ne retrouvons que des arbitres ${ }^{18}$ commerciaux installés comme censeurs des droits nationaux et internationaux (les conventions de l'OIT ${ }^{19}$ ou de la protection de l'environnement signées à Rio et à Kyoto, par exemple).

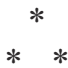

L'enjeu est grave ${ }^{20}$. Les traités sur la protection de l'investissement posent de nombreuses questions sur la politique internationale, le développement de la libéralisation de la société mondiale, le déni de démocratie (puisqu'un AMI, rejeté par les États sous pression des populations, des syndicats, etc, est repris discrètement par un réseau d'ABI offrant aux multinationales les mêmes facilités).

Comme toute action politique, l'imposition des ces règles nouvelles de libéralisation des mouvements du capital est aussi un combat de mots, une lutte pour l'appropriation des mots. Le vocabulaire de la mondialisation est riche de déplacements stratégiques des sens des mots.

Parce que la violence dans les rapports politiques ne peut pas s'exprimer explicitement, elle trouve son chemin, le plus souvent, dans la distorsion du vocabulaire. Cette distorsion permet d'imposer à mots couverts un cadre de pensée et faire passer pour de l'égalité ce qui n'est qu'inégalité, liberté ce qui s'avère contrainte. En somme, lorsqu'il est question de la "protection des investissements de nationaux à l'étranger», ne doit-on pas lire un objectif apparemment moins noble : « offrir la possibilité aux capitaux aujourd'hui investis ici de se délocaliser là où le rendement, le profit sera plus élevé»? ou bien lorsqu'il s'agit «d'offrir une plus grande sécurité aux capitaux pour favoriser l'investissement et donc le développement», ne faut-il pas entendre «donner l'assurance que la main-

18. Pour être désigné comme arbitre selon le CIRDI, il faut avoir une expérience des affaires et, de préférence, une compétence juridique.

19. L'Organisation internationale du travail est un organisme spécialisé des Nations unies. Il est chargé de négocier et de proposer à la ratification des conventions. Il fonctionne suivant le système de la tripartite : gouvernements, syndicats, patronats. En anglais : ILO.

20. Voir J.-C. Deroubaix, M. Robert (dir. C. Gobin), mai 2002, Les accords bilatéraux sur l'investissement dans l'UEBL, rapport de recherche, 44 pages, Bruxelles, Université libre de Bruxelles (Groupe de recherche sur les acteurs internationaux et leurs discours), (texte disponible aussi sur http://users.swing.be/deroubaix). 
d'œuvre locale se laissera exploiter un peu plus que celles des autres pays et abandonnera donc une plus grande part de la valeur produite pour rémunérer ce capital salvateur »?

Le travail sur les mots de la mondialisation, à commencer par ce terme même, doit se poursuivre. Ceci n'est qu'un échantillon. 